\title{
Intensive diabetes management plus coping skills training improved metabolic control and quality of life in adolescents
}

Grey M, Boland EA, Davidson M, et al. Coping skills training for youth with diabetes mellitus has long-lasting effects on
metabolic control and quality of life. J Pediatr $2000 \mathrm{Jul} ; 137: 107-13$.

QUESTION: Is a behavioural programme of intensive diabetes management (IDM) plus coping skills training (CST) more effective than IDM alone for improving metabolic and psychosocial outcomes in adolescents with type 1 diabetes mellitus?

\section{Design}

Randomised (unclear allocation concealment), blinded (clinicians and outcome assessors), controlled trial with 12 months of follow up.

\section{Setting}

A diabetes clinic in New Haven, Connecticut, USA.

\section{Patients}

77 patients who were 12-20 years of age, had no other health problems except for treated hypothyroidism, had been treated with insulin for $\geqslant 1$ year, had a recent glycated haemoglobin concentration $\left(\mathrm{HbA}_{1 \mathrm{c}}\right)$ of $7 \%$ to $14 \%$, had no severe hypoglycaemic events in the previous 6 months, and were in a school grade appropriate to age within 1 year. Follow up was $97 \%$ (mean age $14 \mathrm{y}, 57 \%$ girls, $92 \%$ white).

\section{Intervention}

42 participants were allocated to IDM plus CST and 35 were allocated to IDM alone. IDM comprised $\geqslant 3$ daily insulin injections or continuous subcutaneous insulin infusion, self monitoring of blood glucose concentrations $\geqslant 4$ times daily, monthly outpatient visits, and interim telephone contact. The CST intervention consisted of 6 weekly small group sessions (2-3 participants and a nurse practitioner), each lasting $1-1.5$ hours, and monthly follow up visits for 12 months. The sessions focused on developing coping skills around social problem solving, social skills training, cognitive behaviour modification, and conflict resolution through role playing (eg, managing food choices with friends) with feedback and modelling of appropriate coping behaviour by the nurse practitioner. Follow up role playing with peers continued for $3-5$ weeks to solidify the new behaviours.

\section{Main outcome measures}

Main outcomes included $\mathrm{HbA}_{1 c}$ concentrations, quality of life (Diabetes Quality of Life: Youth scales), self efficacy related to diabetes management (Self-Efficacy for Diabetes Scale), severe hypoglycaemia, and being overweight (body mass index $\geqslant 27.8 \mathrm{~kg} / \mathrm{m}^{2}$ for boys and $\geqslant 27.3 \mathrm{~kg} / \mathrm{m}^{2}$ for girls).

\section{Main results}

At 12 months, patients who received IDM plus CST had lower $\mathrm{HbA}_{1 c}$ concentrations $(7.5 \%$ v 8.5\%, $\mathrm{p}=0.001)$ than patients who received IDM alone, less negative impact of diabetes on quality of life $(\mathrm{p}=0.005)$, and better diabetes self efficacy $(p=0.002)$. Boys in the 2 groups did not differ for episodes of severe hypoglycaemia (27 $v$ $21, \mathrm{p}=0.24)$ nor number who were overweight $(6 v 2$, $p=0.30$ ), whereas girls who received IDM plus CST had fewer episodes of hypoglycaemia $(18 v 22, \mathrm{p}=0.03)$ and fewer were overweight $(1 v 3, \mathrm{p}=0.05)$.

\section{Conclusion}

Adolescents who received intensive diabetes management (IDM) plus a behavioural programme of coping skills training had lower $\mathrm{HbA}_{1 c}$ concentrations and less negative impact of diabetes on quality of life than those who received IDM alone.
Sources of funding: US National Institute of Nursing Research; Culpeper Foundation.

For correspondence: Dr M Grey, Yale University School of Nursing, 100 Church Street South, $P O$ Box 9740, New Haven, CT 06536-0740, USA.Fax $+12037374480$

\section{COMMENTARY}

Adolescents with diabetes have notoriously poor glycaemic control. This follow up study by Grey $e t$ al shows that they can have problems dealing with social situations that may lead to adverse glycaemic effects. It also shows that managing these situations effectively can be learnt with CST. The authors first published their study findings at 6 months follow up and at that time found improvements in $\mathrm{HbA}_{1 \mathrm{c}}$ concentrations, self efficacy, and quality of life. ${ }^{1}$ Unlike most studies that show that the effects of an intervention drop off over time, the beneficial effects of CST persisted at 1 year: glycaemic control in the intervention group continued to be better than that of the control group. A new finding in this follow up study is that, unlike girls, boys in the 2 groups did not differ in hypoglycaemic episodes or being overweight. This may be explained by differences in how boys and girls manage their illness. ${ }^{2}$

The effects on quality of life may initially seem surprising, as one might expect IDM to reduce it. However, the processes of coping and adaptation, facilitated by CST and resulting in increased self efficacy, appear to mitigate the effects of intensive diabetes management on quality of life.

The adolescents studied were predominantly white (95\%) and most were from middle socioeconomic groups; further studies are required to explore the effects of CST on non-white and poorer adolescents. No cost effectiveness analysis was done; such an analysis would be helpful to those charged with deciding whether to implement a similar programme in their clinical settings.

The results are relevant for diabetes educators, clinical nurse specialists, and paediatric nurses. They support the notion that attending to psychosocial outcomes is important, and they also show the dynamic nature of quality of life. From both biomedical and humanistic perspectives, helping adolescents to learn to live and cope with diabetes is probably as important as education.

CST may be a useful adjunct to the armamentarium of tools nurses can use to deal with the psychosocial aspects of diabetes. Its use should not be limited to adolescents; adults too, may benefit from these kinds of behavioural approaches to care.

Maggie Watkinson, RGN, MSc Diabetes Clinical Nurse Specialist Taunton and Somerset NHS Trust

Taunton, Somerset, UK

1 Grey M, Boland EA, Davidson M, et al. Coping skills training for youths with diabetes on intensive therapy. Appl Nurs Res 1999;12:3-12.

2 Williams C. Gender, adolescence and the management of diabetes. J Adv Nurs 1999; 30:1160-6. 